\title{
Comparative clinical and laboratory characteristics of the quality of the filling of root canals using three obturation systems
}

\author{
BR Shumilovich $^{1 *}$, AN Morozov ${ }^{2}$, AV Sushchenko ${ }^{3}$ and D Yu Kharitonov ${ }^{4}$ \\ ${ }^{1}$ Department of Postgraduate Dentistry, Voronezh State Medical University, Russia \\ ${ }^{2}$ Department of Propaedeutic Dentistry, Voronezh State Medical University, Russia \\ ${ }^{3}$ Department of Hospital Dentistry, Voronezh State Medical University, Russia \\ ${ }^{4}$ Department of Surgery Dentistry, Voronezh State Medical University, Russia
}

\begin{abstract}
Universal GuttaFlow System is the first and, up to the present day, only system of cold fluid (plastic) gutta-percha, a unique development of the German company Roeko, a part of the Swiss company Coltene/Whaledent. The material was developed on a base of sealer RoekoSeal that is already well-known to dentists and contains polydimethylsiloxane, which has a property of "reverse capillarity" (sealer fluidity is directly proportional to contraction of volume of space where it is placed). In addition to a sealer, the system contains fragmented gutta-percha $(30 \mathrm{pm})$ and silver microparticles, giving it a number of advantages such as: high fluidity; intracanal expansion during polymerization ( $0,2 \%)$; high biological inertness; automatic mixing system (the material is encapsulated); high antibacterial effect; no need for further condensation.

This allows the material to penetrate easily into dentinal tubules providing a reliable three-dimensional obturation of a system of micro and macro canals during treatment of complicated caries in any clinical situation. The the $\mathrm{X}$-ray evaluations, 3D dental tomography evaluations, scanning electron microscopy and planar elements map was used. In addition, all this allows you to position this material as an obturative system, which is unique in the world for endodontic treatment of different groups of teeth guaranteed with the very high quality.
\end{abstract}

\section{Introduction}

Endodontic treatment of multiroot teeth still represents considerable difficulties for clinicians, in spite of considerable successes in clinical dentistry. Endodontic preparation of canals of this teeth group is complicated in connection with a number of features of their anatomic structure.

These difficulties often lead to the fact that a significant number of dentists at ractical receptions still use root pulp mummification techniques for treatment of complicated caries of multirooted teeth. However, such methods of treatment, unfortunately, do not guarantee a high level of favorable outcomes in the long-term, and drugs used for pulp mummification can have toxic, sensitizing and mutagenic effects. Nowadays, the importance of high-grade quality of instrumental and chemical cleaning of the whole lumen of root canals for any endodontic intervention is universally recognized.

However, the long-term success of conservative endodontic treatment depends not only on the efficiency of cleaning, but also on the reliability of root canal lumen obturation. Hermetic obturation of the canal is a basic guarantee for termination of periodontal tissue antigen stimulation, creation of favorable conditions for reparative processes in periapical areas and prophylaxis of re-infection of periodontium in remote periods after completion of treatment [1-9].

Many methods and techniques for obturation of root canals, each with their own advantages and limitations, were suggested [10]. Filling a root canal with gutta-percha pins (endogenous sealant) and sealer is the most biologically favorable, reliable and durable method in clinical practice. The following basic techniques of root canal fillings are noted: condensation of cold gutta-percha; condensation of cold gutta-percha softened, thermoplastic gutta-percha condensation and many others. Since gutta-percha does not have sufficient adhesiveness and does not stick to canal walls, sealers (endogenous sealants) which provide fitting gutta-percha to edges are needed.

What are the requirements for modern sealers? According to the opinion of many researchers, the ideal sealer should not irritate periodontium, it must tightly obturate the canal in lateral and vertical directions, to prevent shrinkage in the canal and have a bacteriostatic or better bactericidal effect; it must be biocompatible and nontoxic, rentgenocontrast, and must not change teeth color. In addition, the sealer should not be too quick to polymerize, it must have good

Correspondence to: Bogdan R Shumilovich, Voronezh State Medical University, Burdenko, Department of Postgraduate Dentistry, Head of Department. 10, Studencheskaya st., Voronezh, 394036, Russia, Tel: +79-103-449396; E-mail: bogdanshum@gmail.com

Key words: endodontic treatment, root channel obturation, polydimethylsiloxane based siller, cold plastic gutta-percha

Received: October 05, 2015; Accepted: October 24, 2015; Published: October 27,2015 
adhesion both to dentin and obturation material in a root canal after polymerization; it must be insoluble in tissue fluid $[2,4,5,9]$. However, almost none of the sealers used in root canal clinical treatment have all of these properties today.

In connection with the foregoing, sealers created by Roeko (Coltene/ Whaledent, Switzerland, USA) based on polydimethylsiloxane attract some interest. This substance has unique properties of "reverse capillarity", that means the smaller the space in which the sealer is placed, the greater its fluidity. According to the manufacturer, these properties allow the material easily to penetrate into dentinal tubules and obturate them. Creating a system of cold fluid gutta-percha containing fragmented gutta-percha (particle sizes of 30 micrometer) in its composition in addition to the sealer as well as silver micro particles was the next stage of development in this direction [11,12].

However, application of each sealer and/or obturation system in clinical endodontic practice must be based on a comprehensive scientific study of their influence on the state of dental hard tissues, periodontal and above all the quality of obturation of the root canal system not only using conventional techniques, but also on the basis of the results of modern high-tech methods that take into account nuances of both micro structural and biochemical changes at the surface layers of dentin under the influence of sealer $[3,10]$.

The aim of the study was to compare the quality characteristic filling and marginal adaptation and polydimethylsiloxane derivatives most commonly used in clinical practice techniques at the micro and macro levels. Selected for comparison methods are in the same price range as for doctors and patients alike.

\section{Material and methods}

A contingent of 68 people with various nosological forms of complicated caries became the basis for obtaining scientific data consistent with the purpose and objectives of the research. All the patients were divided into 3 groups: a group of gutta-percha lateral compaction, for which obturation of root canals with gutta-percha and the technique of lateral condensation with application of a sealer of Sealpex (Septodont, France) was carried out for the traditional biomechanical preparation; a group of thermoplastic gutta-percha, for which root canal obturation with thermoplastic gutta-percha on Thermafil carrier with application of $\mathrm{AH}$ plus sealer was carried out after the traditional biomechanical training; and a GuttaFlow group, for which obturation of root canals was carried out with the system of cold plastic gutta-percha based on GuttaFlow polydimethylsiloxane without additional application of the sealer and condensation. Only 69 teeth were subjected to endodontic treatment. Anatomical affiliation of teeth in patients of the groups under study is list in Table 1.

Four-level quality control for treatment was carried out in vivo and in vitro for all groups. Standard X-ray control of root canals filling along their lengths (with spot-film radiography, radiovisiography,

Table 1. Anatomical affiliation of the teeth subjected to endodontic treatment at patients of the groups under study.

\begin{tabular}{|l|c|c|c|c|c|}
\hline \multirow{2}{*}{ Group } & \multicolumn{2}{|c|}{ Lower Jaw } & \multicolumn{2}{c|}{ Upper Jaw } & \multirow{2}{*}{ In All } \\
\cline { 2 - 6 } & $\begin{array}{c}\text { Premolar } \\
\text { Teeth }\end{array}$ & $\begin{array}{c}\text { Molar } \\
\text { Teeth }\end{array}$ & Premolar Teeth & $\begin{array}{c}\text { Molar } \\
\text { Teeth }\end{array}$ & In \\
\hline Lateral Compaction & 3 & 9 & 5 & 6 & 23 \\
\hline Thermal Guttapercha & 4 & 5 & 8 & 6 & 23 \\
\hline Guttaflow & 1 & 10 & 8 & 4 & 23 \\
\hline Total & 8 & 24 & 21 & 16 & 69 \\
\hline
\end{tabular}

orthopantomography) and three-dimensional control of root canals filling (with computed 3D tomography), i.e. obturation quality control at the macro level was carried out in vivo.

Studies in vitro were conducted in a specially formed contingent of each group patients. Of these, 5 teeth were removed in patients of the group of gutta-percha lateral condensation (immediately after root canal filling), 5 in patients of the Thermafil group (immediately after root canal filling) and 5 in patients of the group of GuttaFlow patients (immediately after root canal filling). Certainly, as it was more than once mentioned, the main difficulty and interest is connected with endodontic treatment of multiroot teeth, especially molars. For this reason, we in our work have given more attention to this problem.

\section{Results}

Results of endodontic treatments of molars of various anatomic affiliations at patients of a group of lateral compaction are presented in Figures 1-3.

As can be seen from Figures 1-3, X-ray control of the results of endodontic treatment is quite satisfied with all existing current requirements on the quality of root canal fillings along their lengths. 3D computer tomography (Figure $4 \mathrm{a}$ and $4 \mathrm{~b}$ ) was used to study threedimensional Obturation of the root canals.

Violation of three-dimensional fill of the root canals of medial and palatal-buccal roots along their lengths can clearly be seen on Figure $4 \mathrm{a}$ and $4 \mathrm{~b}$. At the same time, violations of three-dimensional filling of distal-buccal canals were not found on any site of cut.

Thus, application of Sealpex sealer in conjunction with the technique of gutta-percha lateral condensation does not provide a guaranteed 3D filling of the root canals along the entire length in all

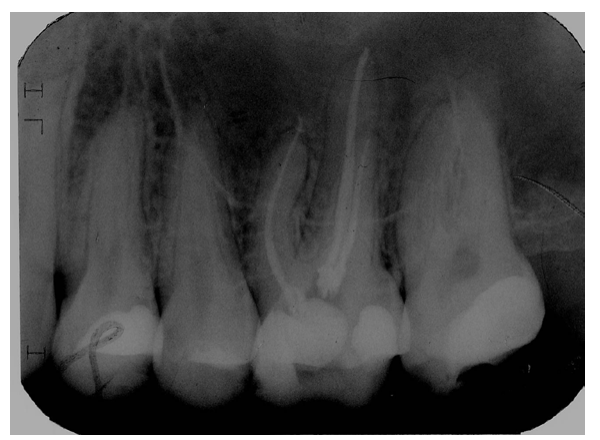

Figure 1. Patient D, age 27. Spot film of tooth 1.6. Method of lateral condensation of guttapercha, sealer Sealpex (treatment result).

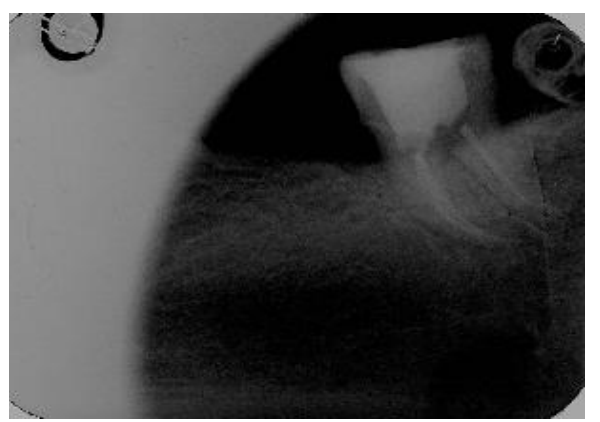

Figure 2. Patient M, age 48. Spot film of tooth 3.7. Method of lateral condensation of guttapercha, sealer Sealpex treatment result). 


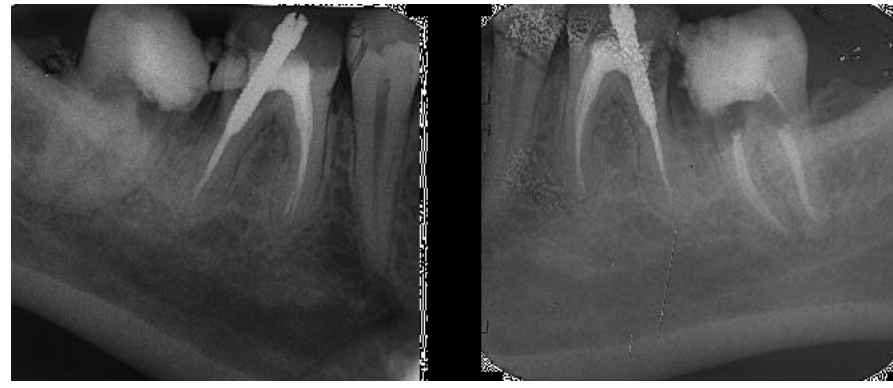

Figure 3. Patient $C$., age 30. Spot film of tooth 4.8. a). Initial clinical situation; b). Treatment result (method of lateral condensation of guttapercha, sealer Sealpex).

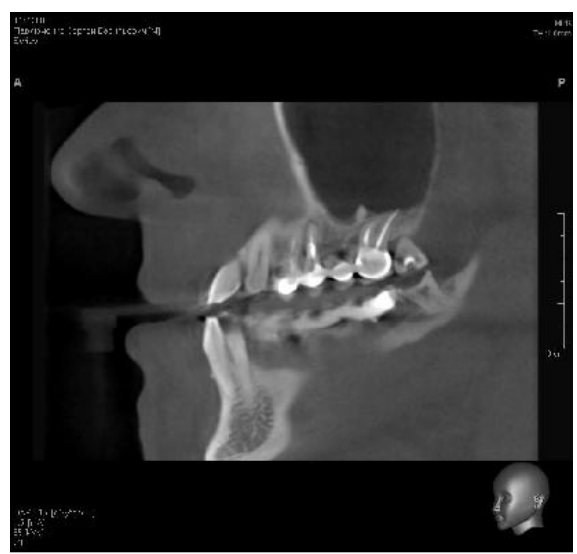

Figure 4a. Patient P., age 46. Fragment of control 3D tomography. Tooth 2.6, method of gutta-percha lateral condensation, sealer Sealpex (treatment result).
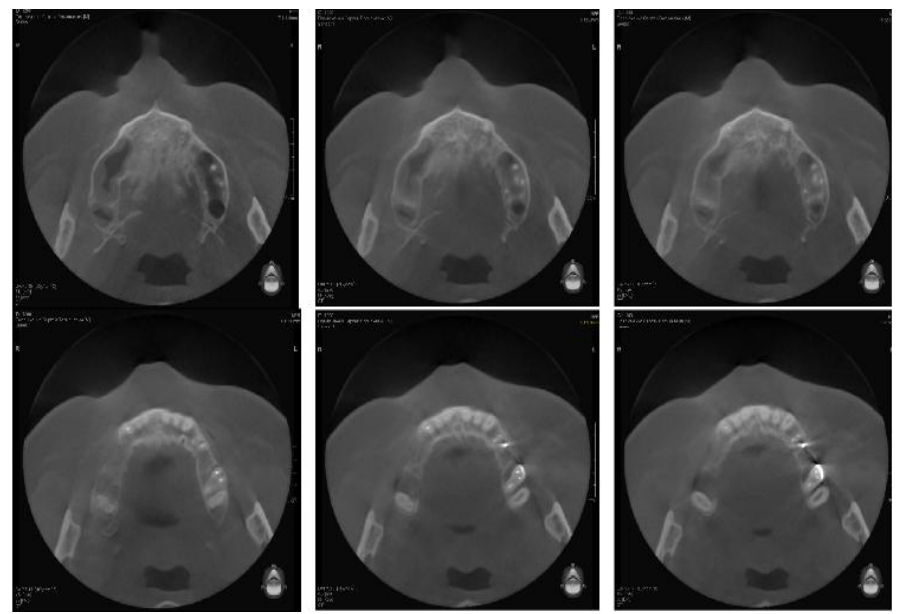

Figure 4b. Patient P., age 46. Fragment of control 3D tomography, sagittal sections of the upper jaw. Tooth 2.6, method of gutta-percha lateral condensation, sealer Sealpex (treatment result).

clinical situations. As we have already mentioned, the results of multirooted teeth treatment, especially premolars and molars, are of keen interest to clinicians. Results of the endodontic treatment of molars with different anatomical affiliation at the Thermafil group patients are shown in Figures 5-7.

As can be seen from Figures 5-7, X-ray control of results of endodontic treatment of patients of this group completely satisfies all existing requirements on the quality of root canal fillings along their length as in previous cases.
Complete three-dimensional filling of root canals over the entire length can be traced in Figure $8 \mathrm{a}$ and $8 \mathrm{~b}$, i.e. the Thermafil system application with $\mathrm{AH}$ plus sealer provides guaranteed 3D filling root canals over the entire length in all clinical situations.

The results of endodontic treatment of molars with different

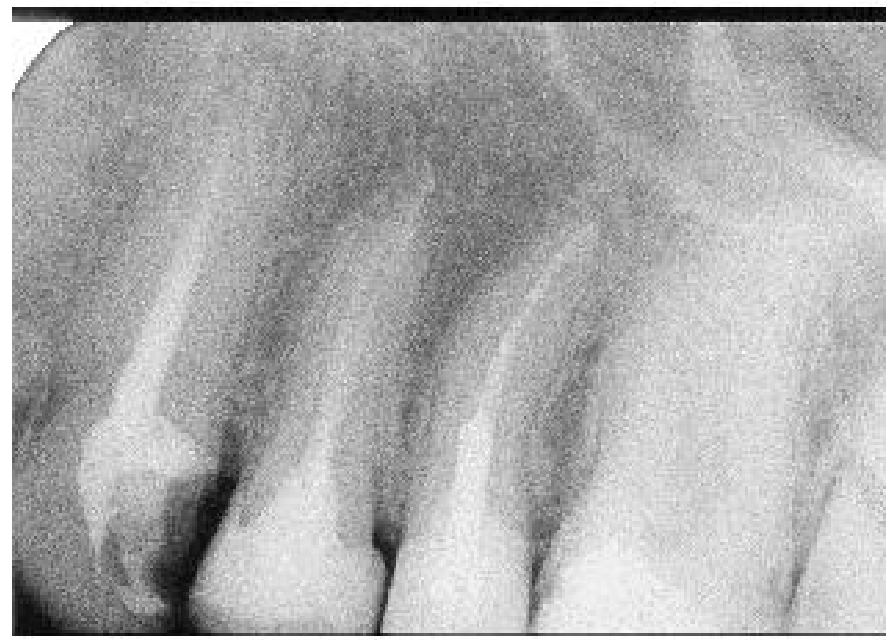

Figure 5. Patient L., age 29. Spot film of teeth 2.4 and 2.5. Thermafil method, sealer AH plus (treatment result).

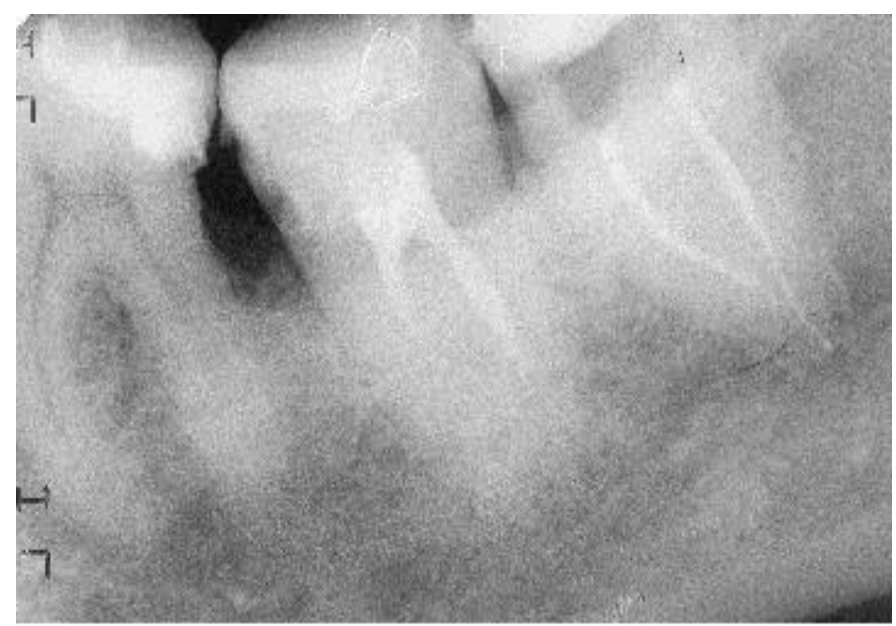

Figure 6. Patient E., age 20. Spot film of tooth 4.8. Thermafil method, sealer AH plus (treatment result).
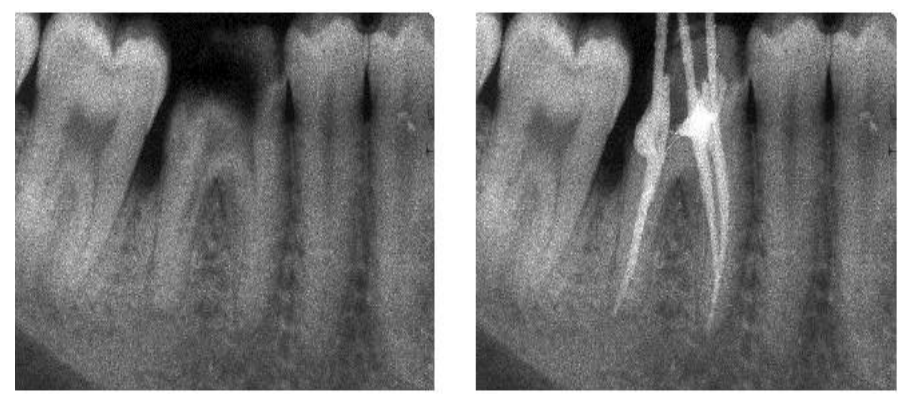

Figure 7. Patient $Z$ h., age 27. Spot film of tooth 2.6. a). Initial situation. b). Treatment result. (Thermafil, AH plus). 


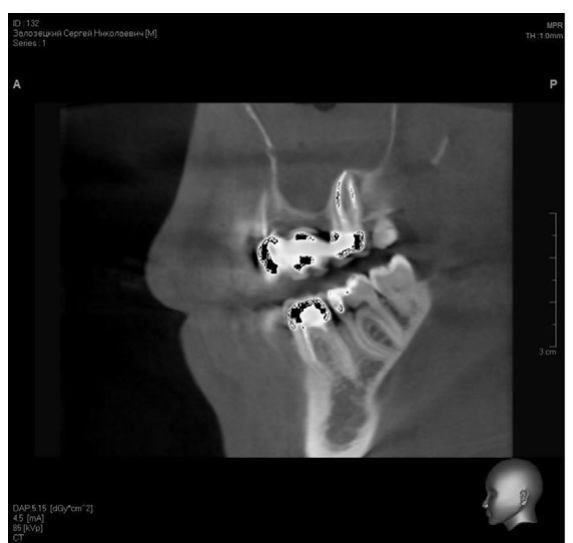

Figure 8a. Patient Z., age 37. Fragment of control 3D tomography. Tooth 2.7, Thermafil method, sealer AH plus (treatment result).
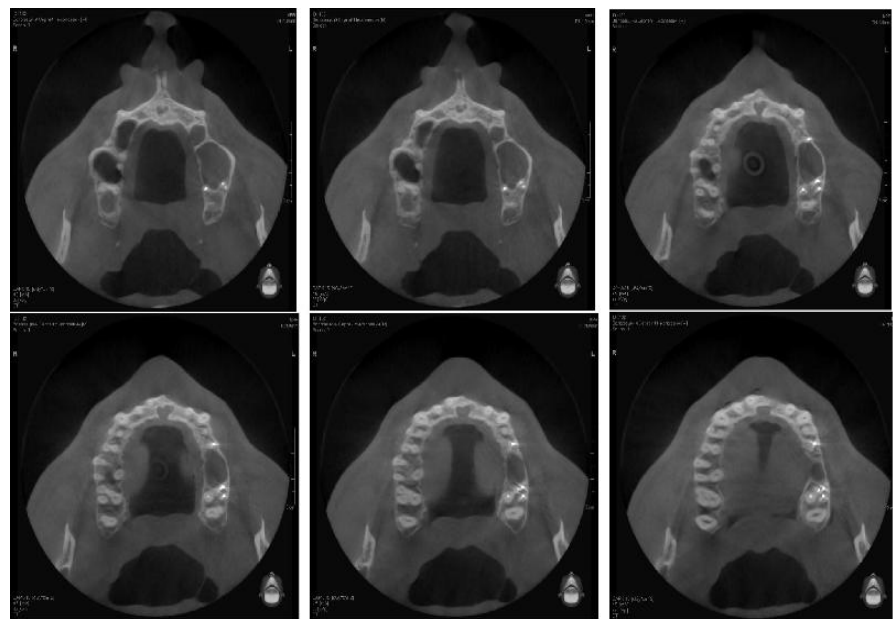

Figure 8b. Patient Z., age 37. Fragment of control 3D tomography, sagittal sections of the upper jaw. Tooth 2.7, Thermafil method, sealer AH plus (treatment result).
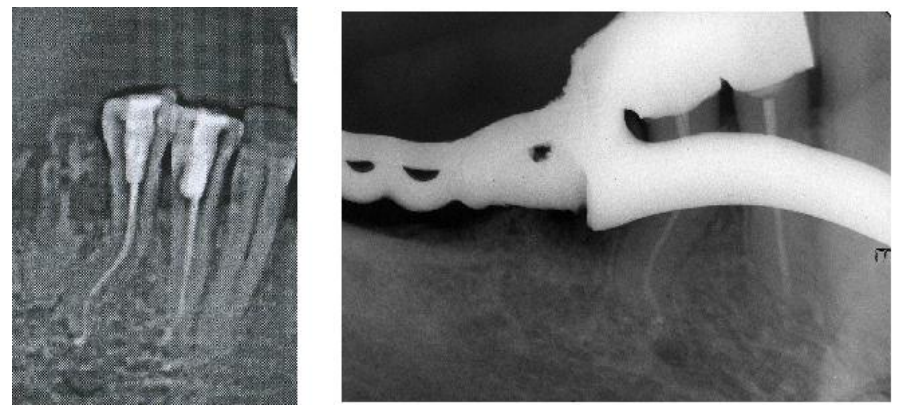

Figure 9. Patient K., age 52. Spot film of teeth 4.4 and 4.5. a). Initial clinical situation. b). Treatment result (GuttaFlow method).

anatomical affiliation with complex root canal anatomy in patients of the GuttaFlow group are shown in Figures 9-13.

As can be seen from Figures 9-11, GuttaFlow provides qualitive, reliable obturation of root canals in teeth with complex root anatomy without additional condensation of the material. GuttaFlow is also successfully used in endodontic treatment of difficult teeth and teeth with resorbed root apex. Initial clinical situation of the 1.8 tooth site (patient B, age 20) is shown in Figure 14a. Control of results of the treatment is shown in Figure 14b.
Thus, as can be seen from Figure 14a and 14b, application of GuttaFlow system, as in the case of application of Thermafil with $\mathrm{AH}$

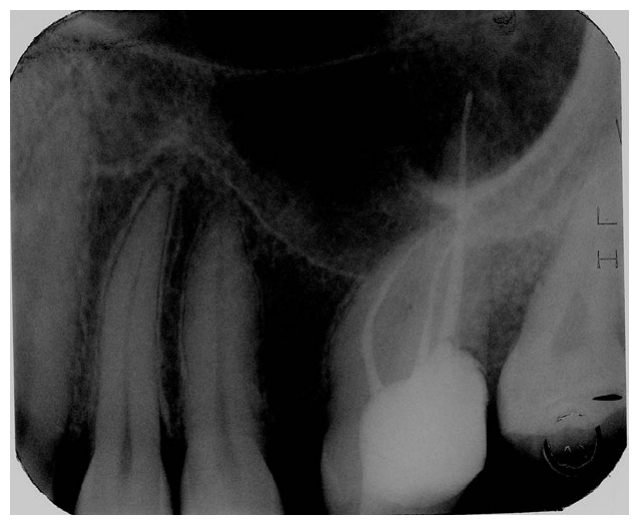

Figure 10. Patient T., age 29. Spot film of tooth 1.7. GuttaFlow method (treatment result).

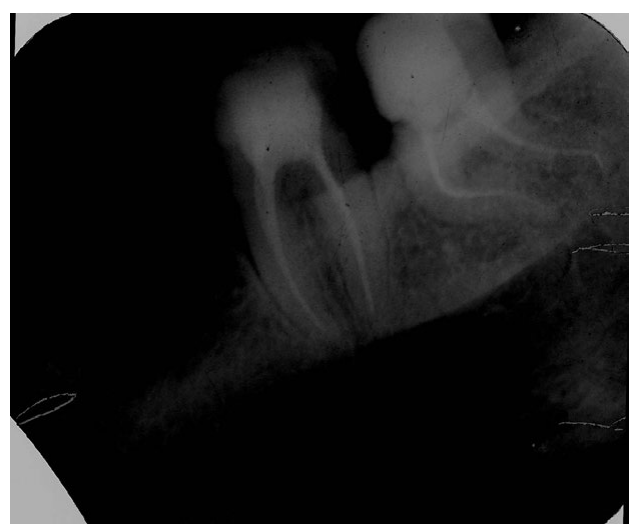

Figure 11. Patient E., age 58. Spot film of teeth 3.7 and 3.8. GuttaFlow method (treatment result).
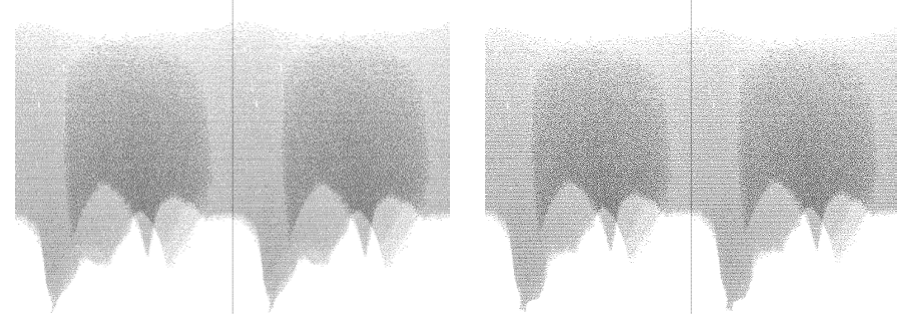

Figure 12. Patient Ts, age 36. Spot film of tooth 3.7. a). Initial clinical situation. b). Treatment result (GuttaFlow method).
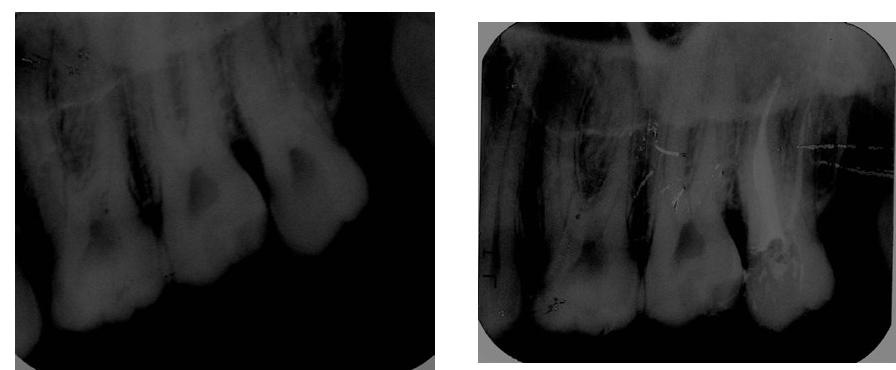

Figure 13. Patient B., age 20. Spot film of tooth 1.8. a). Initial clinical situation. b). Treatment result (GuttaFlow method). 


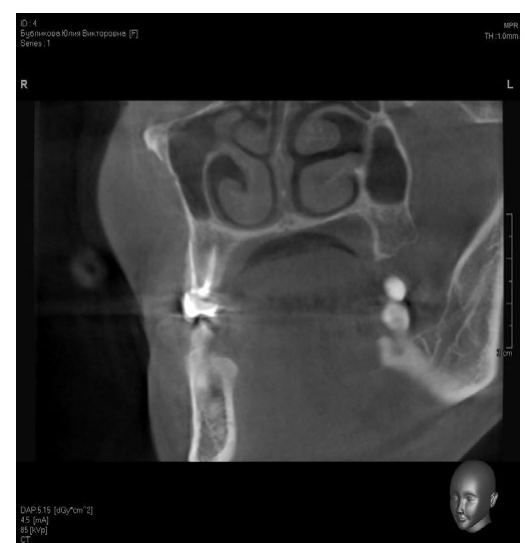

Figure 14 a. Patient B., age 29. Fragment of control 3D tomography. Tooth 1.4, GuttaFlow method (treatment result).
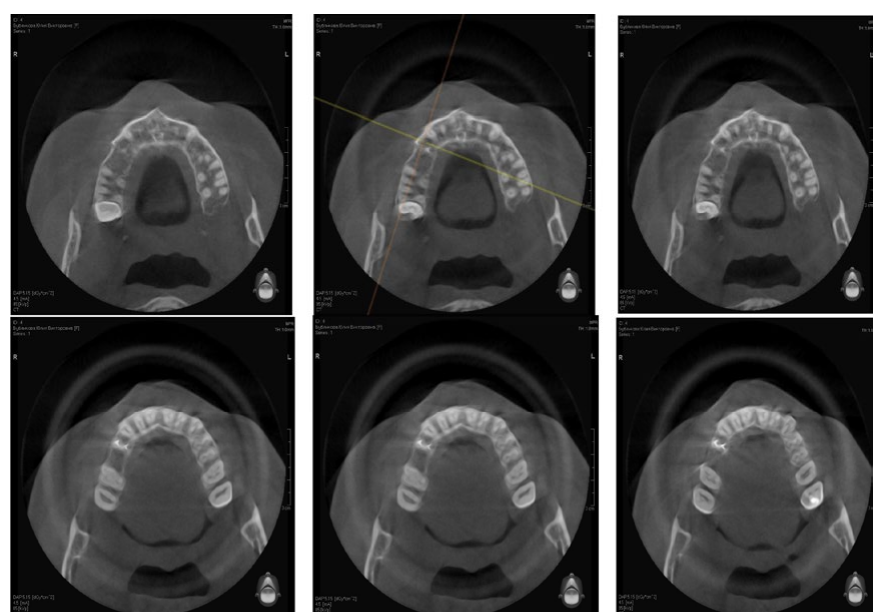

Figure 14b. Patient B., age 29. Fragment of control 3D tomography, sagittal sections of the upper jaw. Tooth 1.4, GuttaFlow method (treatment result).

plus sealer provides guaranteed 3D filling of root canals along the entire length in all clinical situations. Thus, data from clinical research methods register a quite satisfactory quality of filling of root canal length in patients of all groups in case of using traditional methods of control filling (spot-film radiography, radiovisiography) and quality of three-dimensional obturation at patient of the Thermafil group and the GuttaFlow group in case of application of 3D computer tomography.

According to REM, Sealpex sealer, despite the very uneven surface of micro dentin, gutta-percha, provides obturation of the root canal at themicroscopic level in strict compliance of condensation regimes only in its narrow, apical part, where the correct anatomical shape of the latter (Figure 15) was formed. We detected no case of penetration of sealer in the dentinal tubule system as well.

We found presence of 5-10 $\mu \mathrm{m}$ gaps in different sites under study (Figure 16) while scanning the dentin-sealer interface in the root canal coronal part at magnification of X500 and x 1500. In our opinion, giving a clear anatomical form to a root canal is difficult just at these sites; therefore, an adequate condensation is impossible.

Use of SEM allowed determining both structural features of the root dentin adhesive surface and quality of adhesion of the sealer under study to it, as judged by colored planar spectral roentgenograms to assess locations of the studied elements on the interface dentinesealer. Significant concentration of calcium ions at the interface dentinsealer with release of calcium ions into the adhesive "microfissure" is shown in (Figure 17a), which is confirmed by spectrogram marking calcium only (Figure 17b) and indicates absence of airtight occlusion of dentinal tubules, as well as damage of microstructure inside the tubules themselves.

Separately marked phosphorus ions found no changes in its exchange at all studies in patients of all groups (Figure $17 \mathrm{c}$ ). A spectrogram taken at carbon autonomous marking (Figure 17d) shows destruction of dentin organic component.

This characteristic of adhesion quality is displayed most clearly by scanning the surface of root dentin with lateral branches (Figure 18ad). The aforementioned concentration of calcium ions on the interface dentine-sealer is registered on the spectrogram marking calcium only (Figure 18b). The spectrogram taken at carbon autonomous marking

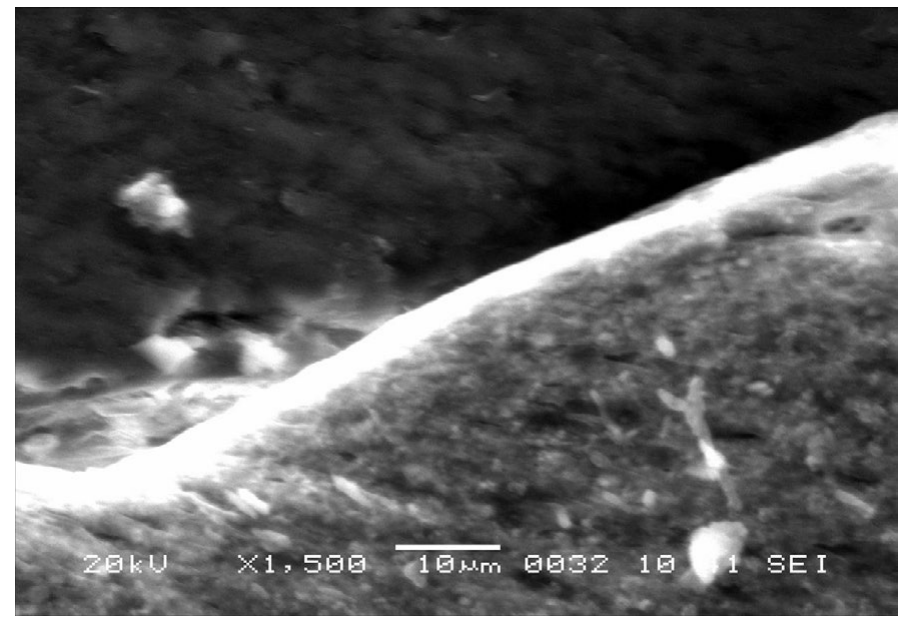

Figure 15. Electron microscopy of the dentin-sealer border at patients of lateral compaction (sealer Sealpex, x1 500, CamScan 4S).

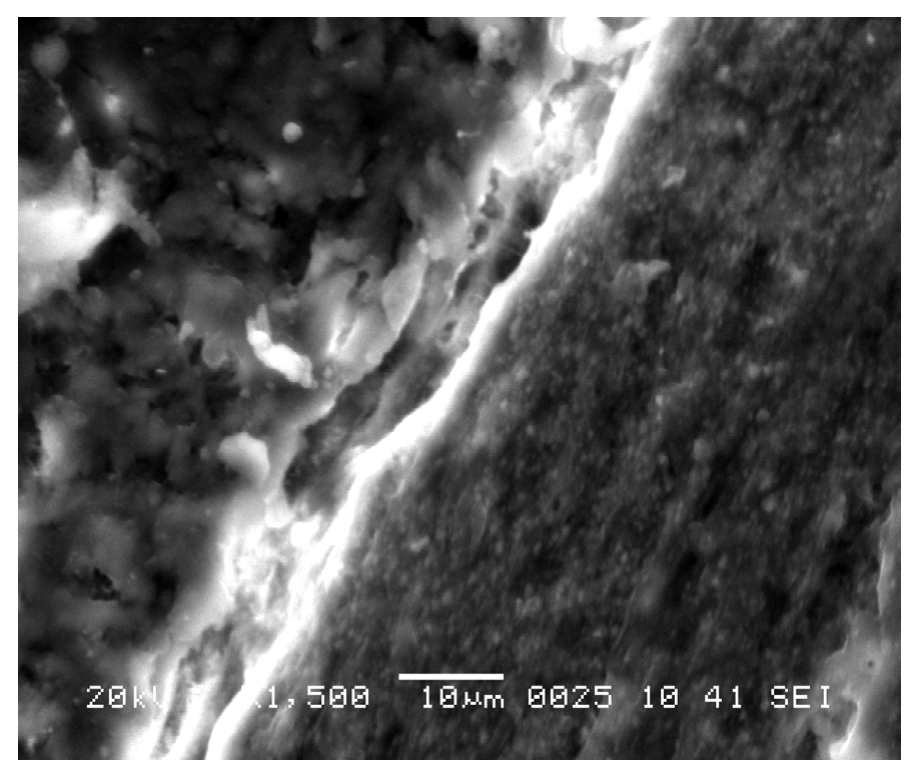

Figure 16. Electron microscopy of the dentin-sealer border at patients of lateral compaction (sealer Sealpex, x1 500, CamScan 4S). 


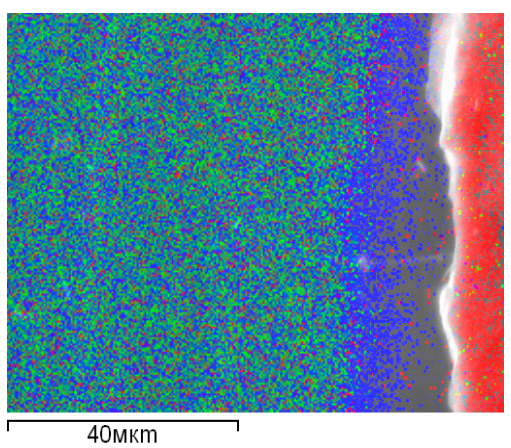

Figure 17a. Planar distribution of chemical elements on the interface dentin-sealer (Sealpex): red - carbon; green - phosphorus; blue - calcium.

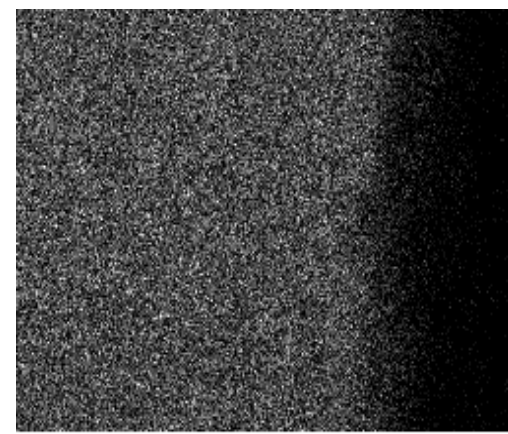

$40 \mathrm{Mkm}$

Figure 17b. Planar distribution of calcium on the interface dentin-sealer (Sealpex).

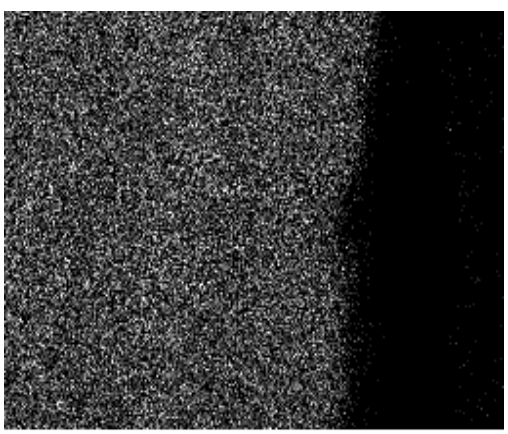

$40 \mathrm{Mkm}$

Figure 17c. Planar distribution of phosphorus on the interface dentin-sealer (Sealpex)

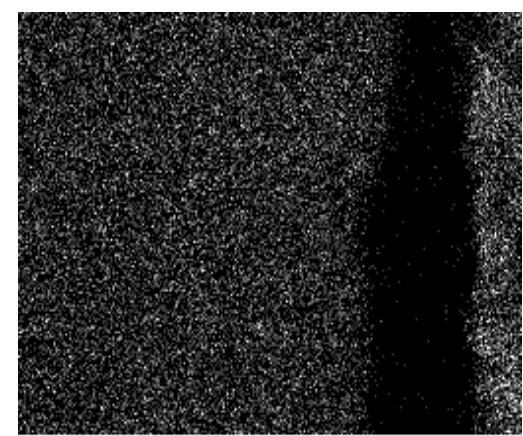

\section{$40 \mathrm{Mkm}$}

Figure 17d. Planar distribution of carbon on the interface dentin-sealer (Sealpex).
(Figure 18d) shows destruction of dentine organic component with a significant accumulation of carbon in the lumen of the dentinal tubule that is not "sealed" with the sealer.

Thus, application of Sealpex sealer even when using a high-quality endodontic instrument does not ensure necessary quality of threedimensional obturation and adhesion as evidenced by presence of "microfissures", absence of penetration of the sealer into dentinal tubules, further destruction of dentin organic component of root canal and loss of calcium that takes place after treatment. Elektronogramma of quality of apical obturation of the root canal in apical constriction is shown in Figure 19. Gutta-percha on a carrier, sealer itself and root

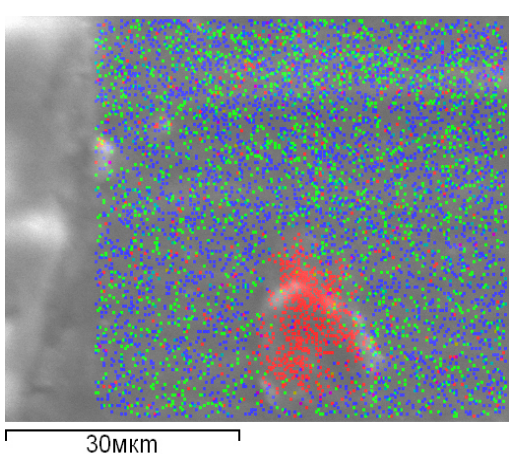

Figure 18a. Planar distribution of chemical elements on the interface dentin-seale (Sealpex): red - carbon; green - phosphorus; blue - calcium.

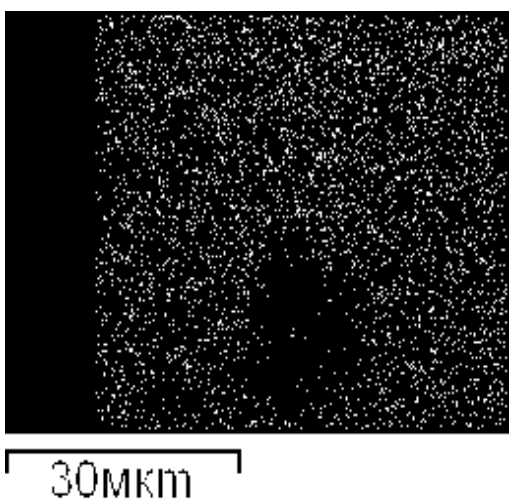

Figure 18b. Planar distribution of calcium on the interface dentin-sealer (Sealpex).

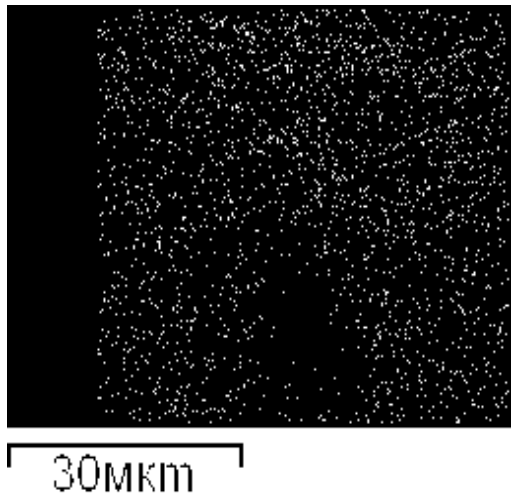

Figure 18c. Planar distribution of phosphorus on the interface dentin-sealer (Sealpex). 


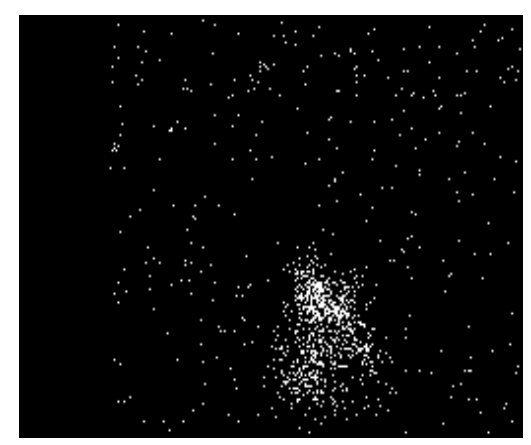

Figure 18d. Planar distribution of carbon on the interface dentin-sealer (Sealpex).

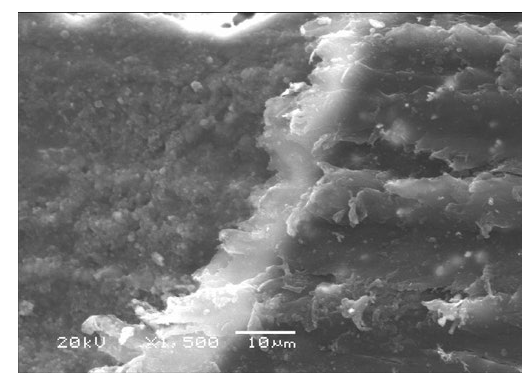

Figure 19. Electron microscopy of the interface dentin-sealer in patients of Thermafil group (x1 500, CamScan 4S).

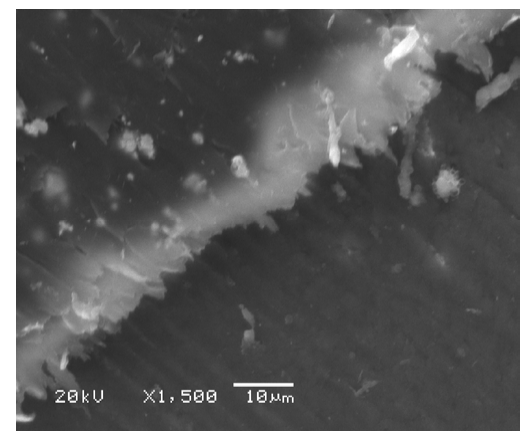

Figure 20. Electron microscopy of the interface dentin-sealer in patients of Thermafil group (x1 500, CamScan 4S).

dentin with microstructure characteristic for apical part are clearly seen in the area of scanning.

We found no damage of the interface dentin-sealer integrity (Figure 20) while scanning it at different sites under study, including those in the root canal coronal part, where giving a clear anatomical form to the root canal was impossible.

Study of obturation quality on a microscopic level in teeth of complex anatomical shape of root canal system (curvature of canals was of $60^{\circ}$ or more) was in this group in a complex of laboratory tests. Elektronogramma of the root canal carried out with magnification x95 is shown in Figure 21a. Such magnification was chosen with a single purpose to place canal lumen along perimeter on the subsequent photograph. The section was performed on a top angle of the root curvature. Shift of the composite carrier located inside Thermafil obturator to direction of the maximum curvature of the root canal (towards the top of the root curvature corner) can clearly be seen in the figure.

"Microfissures" of 10-12 $\mu \mathrm{m}$ size appeared due to mechanical separation of the obturator from the root dentin surface were discovered by us while scanning the area of the "small" curvature of the canal with magnification x1500 (Figure 21b). A compression shift and rupture of the sealer polymer chain with formation of a "microfissure" can clearly be seen in the "big" curvature of the same magnification (Figure 21c).

Construction of colored planar spectral roentgenograms allowing estimated location of the studied elements on the interface dentinesealer (Figure 22a) was carried out in this group. Calcium ions output

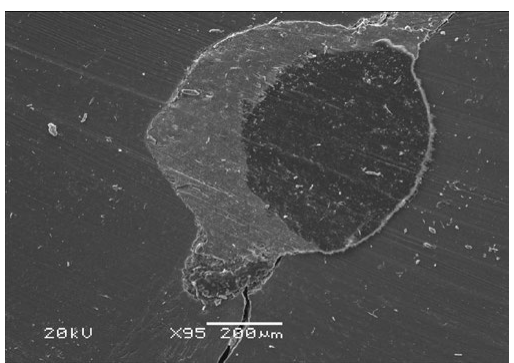

Figure 21a. Electron microscopy of the interface dentin-sealer at patients of Thermafil group (x95, CamScan 4S).

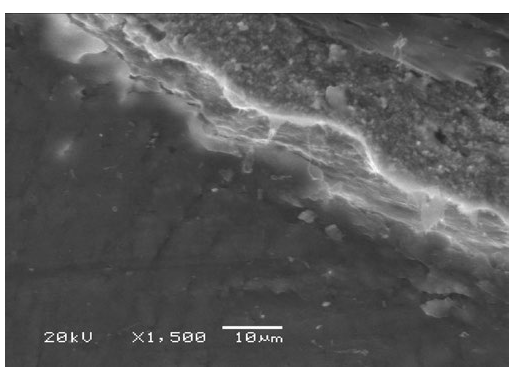

Figure 21b. Electron microscopy of the interface dentin-sealer in a "small" curvature area at patients of Thermafil group (x1 500, CamScan 4S).

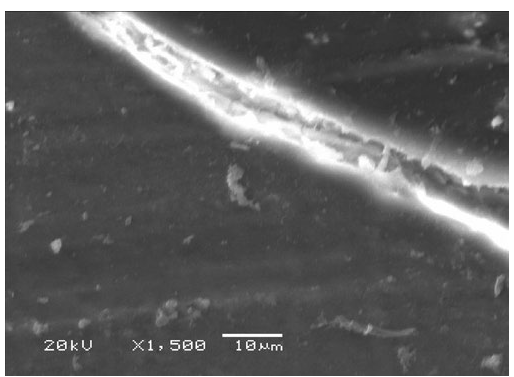

Figure 21c. Electron microscopy of the interface dentin-sealer in a "big" curvature area at patients of Thermafil group (x1 500, CamScan 4S)

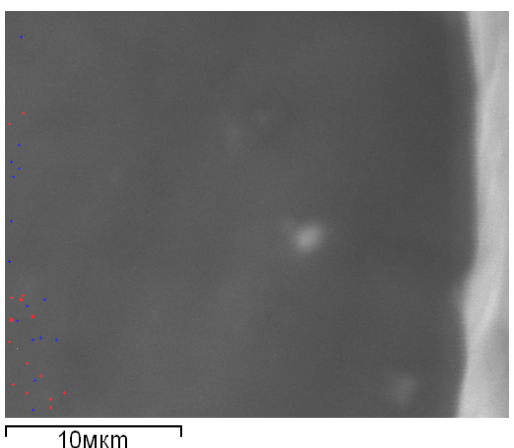

Figure 22a. Planar distribution of chemical elements on the interface dentin-sealer (AH plus, Thermafil); red - carbon; green - phosphorus; blue - calcium. 
at the interface dentin- sealer can clearly be seen on the spectrogram marking calcium only (Figure 22b) and indicates unreliable hermetic obturation of dentinal tubules. The spectrogram taken at carbon autonomous marking (Figure 22d) found no changes in its distribution.

Thus, it can be asserted on the basis of the studies carried out in the Thermafil group that the Thermafil thermoplastic obturator with an $\mathrm{AH}$ plus polymer sealer ensures reliable threedimensional obturation of the root canal only when the curvature angle of the latter does not exceed $60^{\circ}$ and the required taper is created by a biomechanical process. GuttaFlow is equally effective to obturate the root canal in the apical (Figure 23) and crown parts of the root (Figure 24), which is
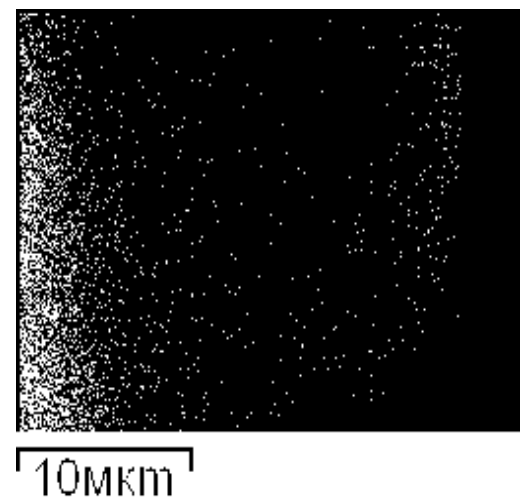

Figure 22b. Planar distribution of calcium on the interface dentin-sealer (AH plus, Thermafil).

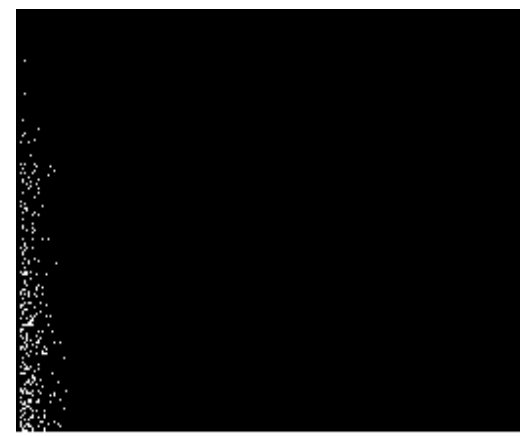

\section{P Ka1}

Figure 22c. Planar distribution of phosphorus on the interface dentin-sealer (AH plus, Thermafil).

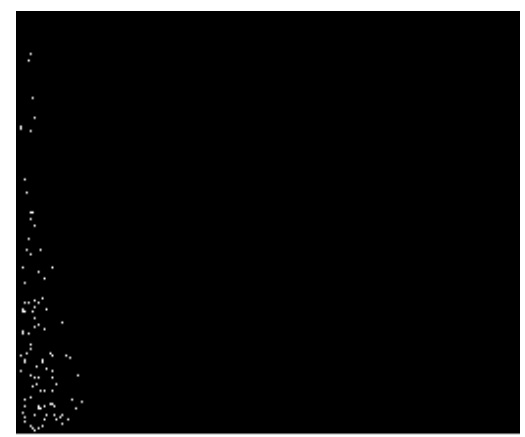

$10 \mathrm{Mk} / 7$

Figure 22d. Planar distribution of carbon on the interface dentin-sealer (AH plus, Thermafil).

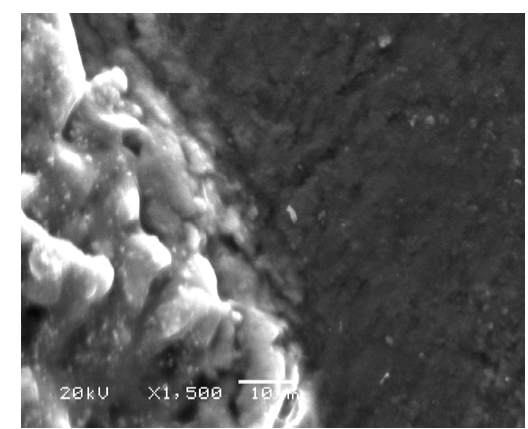

Figure 23. Electron microscopy of the dentin-sealer border at patients of GuttaFlow group (x1500, CamScan 4S).

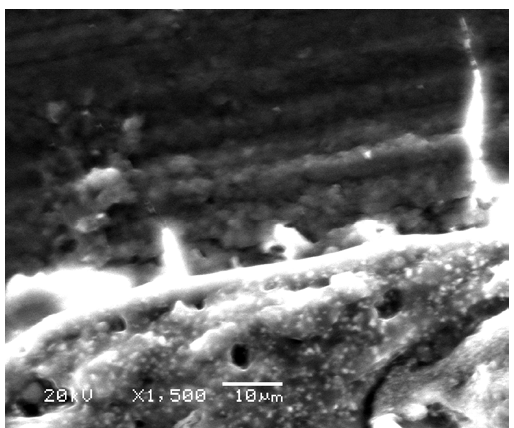

Figure 24. Electron microscopy of the dentin-sealer border at patients of GuttaFlow group (x1500, CamScan 4S)

clearly displayed when you scan the interface dentin-sealer in different sites of research including the crown root canal where giving a clear anatomical form to the root canal was impossible.

Elektronogramma of the quality of apical obturation of the root canal in apical constriction is shown in Figure 23. Gutta-percha filling with the sealer penetrating into the system of micro canals and the root dentin itself with a microstructure characteristic for the apical part is clearly seen in the area of scanning.

In spite of a very heterogeneous micro relief of dentin surface, the GuttaFlow system in all studies performed in this group at a microscopic level provided a reliable three-dimensional obturation of root canals with penetration into dentinal microtubules and guaranteed them a reliable obturation.

Obturation quality study at the microscopic level in teeth with complex anatomical shape of the root canal system (curvature of the canal was more than $60^{\circ}$ ) was conducted for comparison with data received in the Thermafil group during complex laboratory researches.

Elektronogramma of the root canal performed with magnification $\mathrm{x} 110$ is shown in Figure 25a. Such magnification was chosen with a single purpose to place canal lumen along the complete perimeter in the subsequent photograph. The section was performed on a top angle of the root curvature. Even filling of the root canal lumen with guttapercha filling of the system around the complete perimeter without shifting aside can clearly be seen in the figure, which indicates absence of mechanical tension of obturation at this area.

We have found integrity of the interface filling-dentin without "microfissures" and mechanical separation of filler from the root dentin surface (Figure 25b) by scanning the area of the canal "small" curvature with magnification x1500. The situation in the area of "high" 


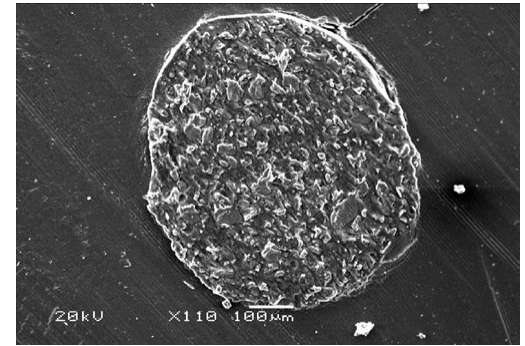

Figure 25a. Electron microscopy of the dentin-sealer border in patients of GuttaFlow group (x1500, CamScan 4S).

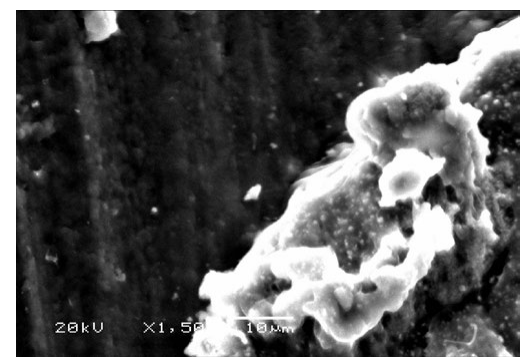

Figure 25b. Electron microscopy of the interface dentin-sealer in a "small" curvature area in patients of GuttaFlow group (x1500, CamScan 4S).

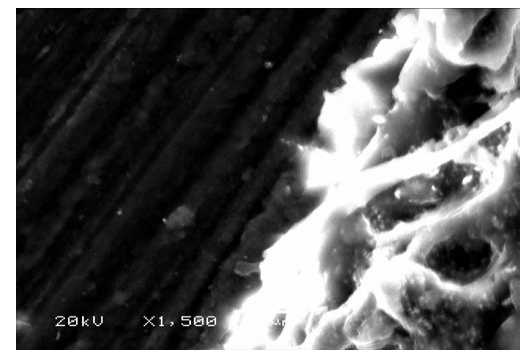

Figure 25c. Electron microscopy of the interface dentin-sealer in a "big" curvature area in patients of GuttaFlow group (x1 500, CamScan 4S).

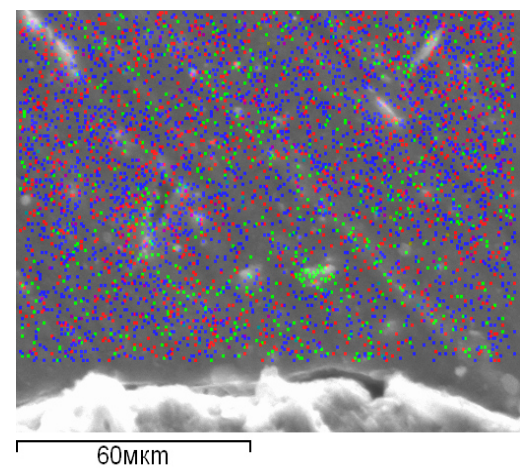

Figure 26a. Planar distribution of chemical elements on the interface dentin-sealer (GuttaFlow); red - carbon; green - phosphorus; blue - calcium.

curvature is shown in Figure 25c. Integrity of the interface filler-dentin is clearly seen also under magnification x 1500 .

Construction of colored planar spectral roentgenograms allowing estimated location of the studied elements on the interface dentinesealer (Figure 26a) was carried out with SEM use. Integrity of the studied interface with absence of calcium ions output can clearly be seen on the spectrogram, marking only the calcium (Figure 26b) that indicates high tightness of obturation of the dentinal tubules system. Separately marked phosphorus ions found no changes in its metabolism like in all groups under study (Figure 26c). The spectrogram taken at carbon autonomous marking (Figure 26d) also found no changes in its distribution.

Thus, it can be argued on the basis of the studies carried out in the GuttaFlow group that the system of cold fluid gutta-percha with the sealer based on polydimethylsiloxane included in the composition is highly effective and ensures reliable three-dimensional obturation of the root canal and a system of dentinal micro channels in all clinical situations that were confirmed by radiography and computed

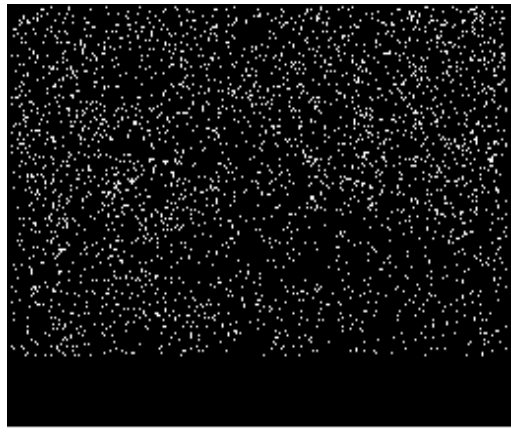

$60 \mathrm{Mkm}$

Figure 26b. Planar distribution of calcium on the interface dentin-sealer (GuttaFlow)

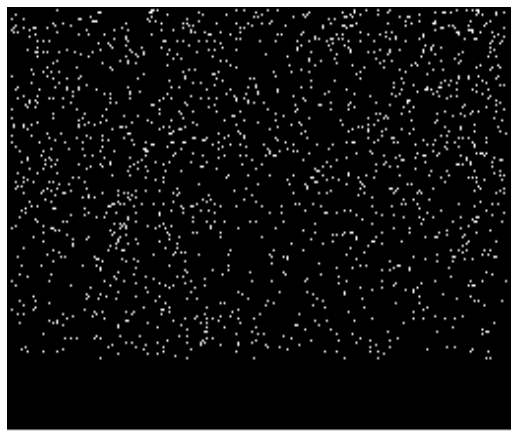

\section{P Ka1}

Figure 26c. Planar distribution of phosphorus on the interface dentin-sealer (GuttaFlow)

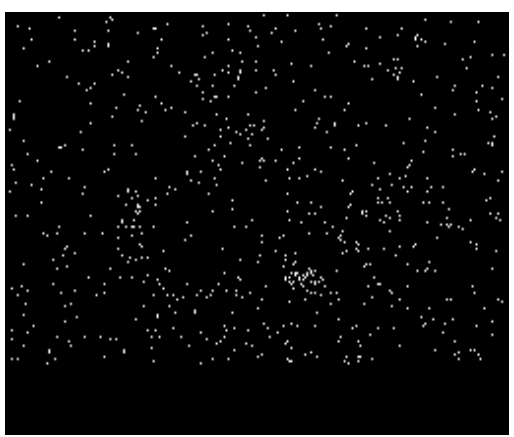

Figure 26d. Planar distribution of carbon on the interface dentinsealer (GuttaFlow). 
tomography. As for other obvious advantages of this system, it should be noted not only absence of shrinkage and even a slight expansion, as well as high speed, unique fluidity of material, ease of introduction into root canals, high biological inertness to periodontal tissues, possibility of micro canal obturation, as well as antibacterial properties, which are pronounced due to the presence of silver nanoparticles and occur without causing posterior discolorations. The main advantage of all systems that are used without further gutta-percha condensation inherent to this system: the required quality of obturation is provided even in cases where anatomic form of a canal prepared for filling is far from ideal. And, accurate observance of the canal conycity is not an indispensable condition to use of the system of GuttaFlow.

Necessity of acquisition of additional equipment and a long time of exposition 30-40 minutes can be considered as relative disadvantages of this system, though now a GuttaFlow Fast system with time of exposition of 15 minutes already exists in the market. Thus, use of the complex techniques allows estimating various physiological indicators of hard tooth tissues and yields a possibility to track a number of laws in the processes proceeding in the root canal dentin under influence of various kinds and its obturation methods during a complicated caries treatment $[6,7]$.

Per our data, all the sealers under study provide admissible quality of obturation of root canals on macro- and micro-levels, but the best results of quality endodontic treatments on macro, micro and biochemical levels are received only after application of the GuttaFlow system.

From the aforesaid it follows that now dentists treating complicated caries use various techniques of biomechanical preparation and obturation of root canals which choice depends, first of all, on a qualitative level of a dentistry clinic, its financial possibilities, the cultural level of population in a country and a creative approach by the medical staff. In these conditions, it is required to prove accurately economic and medical necessity of application of new innovative methods of diagnostics and treatment, which should be used, certainly, not for "surroundings" or exclusively marketing purposes, but to serve the basic aim - to improve the quality of stomatologic help, with the greatest decrease in the number of complications and relapses. Taking into consideration the fact that modern clinical endodontic passes to the principle of minimum invasive interventions at biomechanical preparation when canal conicity and correct anatomic form is impossible, it is possible to tell with confidence, that inclusion of sealers on the basis of polydimethylsiloxane, especially of the GuttaFlow system, into medical tactics of doctor-dentists at endodontic treatment allows for desirable results.

\section{References}

1. Bouillaguet S, Shaw L, Barthelemy J, Krejci I, Wataha JC (2008) Long-term sealing ability of Pulp Canal Sealer, AH-Plus, GuttaFlow and Epiphany. Int Endod J 41: 219226. [Crossref]

2. Brzovic V, Miletic I, Zeljezic D, Mladinic M, Kasuba V, et al. (2009) In vitro genotoxicity of root canal sealers. Int Endod J 42: 253-263. [Crossref]

3. Elayouti A, Achleithner C, Löst C, Weiger R (2005) Homogeneity and adaptation of a new gutta-percha paste to root canal walls. J Endod 31: 687-690. [Crossref]

4. Eldeniz AU, Ørstavik D. 2654 Five New Endodontic Sealers' In Vitro Resistance to Bacterial Penetration. IADR/AADR/CADR 85th General Session and Exhibition (March 21-24, 2007), New Orleans.

5. Hammad M, Qualtrough A, Silikas N (2009) Evaluation of root canal obturation: a three-dimensional in vitro study. J Endod 35: 541-544. [Crossref]

6. Herbert J, Bruder M, Braunsteiner J, Altenburger MJ, Wrbas KT (2009) Apical quality and adaptation of Resilon, EndoREZ, and Guttaflow root canal fillings in combination with a noncompaction technique. J Endod 35: 261-264. [Crossref]

7. Kiefner P, ElAyouti A, Hecker H, Löst C, Weiger R (2007) Influence of four different obturation techniques on the quality of gutta-percha root fillings. Int Endod J 40: 999.

8. Kqiku L, Städtler P, Gruber HJ (2008) Dichtigkeit von Wurzelkanalfüllungen mit GuttaFlow und Resilon im Vergleich zur lateralen Kondensation. Stomatologie September; 105: 107.

9. Mohammadi Z, Yazdizadeh M (2007) Evaluation of the antibacterial activity of new root canal sealers. J Clin Dent 18: 70-72. [Crossref]

10. Ozok AR, van der Sluis LW, Wu MK, Wesselink PR (2008) Sealing ability of a new polydimethylsiloxane-based root canal filling material. J Endod 34: 204-207. [Crossref]

11. Sumilovich B, Naumova A, Kholodovich O (2011) Quality control of root channels obturation in use polydimethylsiloxane based sillers. Endodonty Today 3: 72-76.

12. Zielinski TM, Baumgartner JC, Marshall JG (2008) An evaluation of Guttaflow and gutta-percha in the filling of lateral grooves and depressions. $J$ Endod 34: 295-298. [Crossref]

Copyright: (C2015 Shumilovich BR. This is an open-access article distributed under the terms of the Creative Commons Attribution License, which permits unrestricted use, distribution, and reproduction in any medium, provided the original author and source are credited. 